\title{
Cardiovascular imaging 2020 in the international journal of cardiovascular imaging: the 10 most downloaded papers in the year 2020
}

\author{
Arthur Stillman ${ }^{1}$. Johan H.C. Reiber ${ }^{2}$
}

Published online: 30 March 2021

(c) The Author(s), under exclusive licence to Springer Nature B.V. 2021

The international Journal of Cardiovascular Imaging had 258,168 downloads of manuscripts in 2020. This is increased from 2019 which had the previous most downloads at 186,182 .

The most frequently downloaded manuscript was "Angiography-derived index of microcirculatory resistance as a novel, pressure-wire-free tool to assess coronary microcirculation in ST elevation myocardial infarction" by De Maria et al. [1]. This manuscript was downloaded 11909 times. This paper developed and validated an angiography derived index of microvascular resistance as a novel pressure-wirefree index of microvascular resistance.

"Right ventricular free wall longitudinal strain and strain rate quantification with cardiovascular magnetic resonance based tissue tracking" by Qu et al. [2] was downloaded 1755 times. This paper established reference values of right ventricular free wall regional and segmental peak strain rates and investigated gender and age- related differences.

"Left atrial ejection fraction and outcomes in heart failure with preserved ejection fraction" by Kanagala et al. [3] was downloaded 1358 times. These authors found worse prognosis for patients with preserved ejection fraction and heart failure when the left atrial ejection fraction is diminished.

"Cardiac imaging procedures and the COVID-19 pandemic: recommendations of the European Society of Cardiovascular Radiology (ESCR)" by Beitzke et al. [4] was downloaded 1180 times. Recommendations were made for cardiovascular imaging during the pandemic in both noninfected and COVID-19 patients.

Johan H.C. Reiber

J.H.C.Reiber@lumc.nl

1 Department of Radiology and Imaging Sciences, Atlanta, USA

2 Department of Radiology, Leiden University Medical Center, Leiden, The Netherlands
"Comparison of quantitative flow ratio and fractional flow reserve with myocardial perfusion scintigraphy and cardiovascular magnetic resonance as reference standard. A DanNICAD substudy" by Sejr-Hansen et al. [5] was downloaded 1087 times. These workers compared $\mathrm{FFR}_{\mathrm{CT}}$ and CT perfusion (CTP) to FFR. FFR $\mathrm{CT}_{\mathrm{C}}$ was found to have superior diagnostic accuracy to visually and semi-quantitatively assessed static rest/stress CTP.

"Effects of baseline heart rate at sea level on cardiac responses to high-altitude exposure" by Tian et al. [6] was download 932 times. These workers found that high altitude was associated with an increase of heart rate and decrease in stroke volume index preserving the cardiac output index.

"A multi-vendor, multi-center study on reproducibility and comparability of fast strain-encoded cardiovascular magnetic resonance imaging" by Erley et al. [7] was downloaded 892 times. These authors studied the inter-vender agreement and test-retest reproducibility of fast strainencoding acquisitions at $3 \mathrm{~T}$ on 15 volunteers and found good inter-vendor agreement and test-retest reproducibility providing the basis for conducting studies to validate strain techniques in large patient cohorts.

“"'Pure" severe aortic stenosis without concomitant valvular heart diseases: echocardiographic and pathophysiological features" by Kandels et al. [8] was downloaded 817 times. This provocative study found that "pure" aortic stenosis defined as an indexed effective orifice area $<0.6$ $\mathrm{cm}^{2}$ accepted pathophysiological sequela of left ventricular hypertrophy, diastolic dysfunction and pulmonary artery hypertension cannot be confirmed.

"In-vitro and in-vivo imaging of coronary artery stents with Heartbeat OCT" by Cecchetti et al. [9] was downloaded 767 times. These authors studied the impact of cardiac motion on stent length measurements with optical coherence tomography and demonstrated motion artifact free OCT imaging of implanted stents using ECG triggering and rapid pullback. 
"Echocardiographic features of patients with COVID-19 infection: a cross-sectional study" by Barman et al. [10] was downloaded 758 times. These authors found that both right and left ventricular function were diminished with more severe disease.

These papers were the most frequently downloaded manuscripts last year. There were many more excellent contributions in 2020 which can be expected to be cited in the coming years.

Arthur Stillman, MD, PhD: Associate Editor

Johan HC Reiber, PhD: Editor-in-Chief

\section{References}

1. De Maria GL, Scarsini R, Shanmuganathan M, Kotronias RA, Terentes-Printzios D, Borlotti A et al (2020) Angiography-derived index of microcirculatory resistance as a novel, pressure-wire-free tool to assess coronary microcirculation in ST elevation myocardial infarction. Int J Cardiovasc Imaging 107:3129-12. https://doi. org/10.1007/s10554-020-01831-7

2. Qu Y-Y, Li H, Rottbauer W, Ma G-S, Buckert D, Rasche V (2020) Right ventricular free wall longitudinal strain and strain rate quantification with cardiovascular magnetic resonance based tissue tracking. Int J Cardiovasc Imaging 36:1985-1996. https://doi. org/10.1007/s10554-020-01895-5

3. Kanagala P, Arnold JR, Cheng ASH, Singh A, Khan JN, Gulsin GS et al (2020) Left atrial ejection fraction and outcomes in heart failure with preserved ejection fraction. Int J Cardiovasc Imaging 36:101-110. https://doi.org/10.1007/s10554-019-01684-9

4. Beitzke D, Salgado R, Francone M, Kreitner K-F, Natale L, Bremerich J et al (2020) Cardiac imaging procedures and the
COVID-19 pandemic: recommendations of the European Society of Cardiovascular Radiology (ESCR). Int J Cardiovasc Imaging 36:1801-1810. https://doi.org/10.1007/s10554-020-01892-8

5. Sejr-Hansen M, Westra J, Winther S, Tu S, Nissen L, Gormsen L et al (2020) Comparison of quantitative flow ratio and fractional flow reserve with myocardial perfusion scintigraphy and cardiovascular magnetic resonance as reference standard. A DanNICAD substudy. Int J Cardiovasc Imaging 36:395-402. https:// doi.org/10.1007/s10554-019-01737-Z

6. Tian J, Liu C, Yang Y, Yu S, Yang J, Zhang J et al (2020) Effects of baseline heart rate at sea level on cardiac responses to highaltitude exposure. Int J Cardiovasc Imaging 36:799-810. https:// doi.org/10.1007/s10554-020-01769-w

7. Erley J, Zieschang V, Lapinskas T, Demir A, Wiesemann S, Haass $\mathrm{M}$ et al (2020) A multi-vendor, multi-center study on reproducibility and comparability of fast strain-encoded cardiovascular magnetic resonance imaging. Int J Cardiovasc Imaging 36:899-911. https://doi.org/10.1007/s10554-020-01775-y

8. Kandels J, Tayal B, Hagendorff A, Lavall D, Laufs U, Sogaard P et al (2020) "Pure" severe aortic stenosis without concomitant valvular heart diseases: echocardiographic and pathophysiological features. Int J Cardiovasc Imaging 36:1917-1929. https://doi.org/ 10.1007/s10554-020-01907-4

9. Cecchetti L, Wang T, Hoogendoorn A, Witberg KT, Ligthart JMR, Daemen J et al (2020) In-vitro and in-vivo imaging of coronary artery stents with Heartbeat OCT. Int J Cardiovasc Imaging 36:1021-1029. https://doi.org/10.1007/s10554-020-01796-7

10. Barman HA, Atici A, Tekin EA, Baycan OF, Alici G, Meric BK et al (2020) Echocardiographic features of patients with COVID19 infection: a cross-sectional study. Int J Cardiovasc Imaging. https://doi.org/10.1007/s10554-020-02051-9

Publisher's Note Springer Nature remains neutral with regard to jurisdictional claims in published maps and institutional affiliations. 